\title{
Collaborative Robots: Frontiers of Current Literature
}

\author{
Mikkel Knudsen $^{1}$ (D) Jari Kaivo-Oja ${ }^{1}$ \\ ${ }^{1}$ Finland Futures Research Centre, University of Turku, Finland
}

\begin{abstract}
Collaborative Robots (Cobots) play an increasing role in the advanced manufacturing landscape. The cobot market is rapidly expanding, and the academic literature is similarly growing. This paper presents a short overview of current cobotic trends and futures frontiers of the cobot development with particular emphasis on the role of cobots within the Industry 4.0-paradigm and within global megatrends. Since there is a strange, but distinct lack of literature reviews on the topic of collaborative robotics, the paper provides novel and valuable insights. In highlighting current frontiers, the paper also illustrates potential developments of future human-robot interaction.
\end{abstract}

Keywords: Collaborative Robots, Cobots, Human-machine interaction, Industry 4.0

\section{Introduction}

Collaborative robots or "cobots" increasingly enter industrial manufacturing, and academics, practitioners, and market followers have started to take note. Cobot technology can be a game-changer and the dominant robot technology in the decades to come (Bloss, 2016), and collaborative robotics has already become one of the fastest-growing sectors of the robotics market (Goldberg, 2019).

The evolution of robots, and particularly of cobots, have been tremendous over the last ten years (FastBerglund et al., 2016; Hentout et al., 2019).

Collaborative robots mark a departure from traditional industrial robots which functions separated from their human co-workers. Cobots, on the other hand, are designed for direct interaction with human workers, to handle shared payload, and to function safely without conventional safety cages or similar protective measures (Peshkin \& Colgate, 1999; Bogue, 2016). In the words of Korn et al. (2018), "robots have long left the cages of industrial settings: They work together with humans collaboratively". This collaboration leverages the flexibility and decision making of humans with the strength and endurance of robots with (Djuric et al., 2016). The majority of cobots are lightweight and possible to move between places, i.e. they are, in the passive sense at least, mobile (Bendel, 2018).

This makes cobots a good fit as a gateway into factory automation (Marvel, 2014) and as an enabling technology for the so-called Fourth Industrial
Revolution in which flexibility is a critical component supplementing automation.

This article provides a short literature-based state-ofthe-art overview of cobot developments. Until now, this kind of overview is strangely lacking from academic literature, wherefore this article provides important contributions within this rapidly growing field.

The article first provides a glimpse of the current market trends as well as an introduction to the academic literature on cobots. The second subchapter positions cobots within ongoing changes to manufacturing paradigms and the new Industry 4.0, before the article touches upon cobots' potential role within current global megatrends. Based on the literature the authors then present current frontiers of collaborative robotics along three main dimensions: Economic, social, and technological. These frontiers provide valuable insights and signals for possible futures of cobots over the next decades.

\section{The growing interest in cobots}

\subsection{Academic literature}

The main source of information for this article is the international academic publication database Web of Science. A literature search has been performed in the database for "collaborative robot*" or "cobot" to appear either in the title of publications or as a keyword. The literature search provided a total of 795 articles on cobots by January 22nd, 2020.

\footnotetext{
* Corresponding Author. Phone: +358505136636

E-mail: mikkel.knudsen@utu.fi
} 
In Figure 1, we have outlined the growing amount of publications on cobot technology available in Web of Science. The first article in the database was published in 1996, but it took until 2014 to pass more than 20 published cobot-articles in a single year, still with a relatively meager 22 publications. This grew to more than 100 articles in 2017 (total 119), before a new single-year record of 193 publications in 2019. As the figure shows, we have moved to a very strong growth phase of academic interest in the topic.

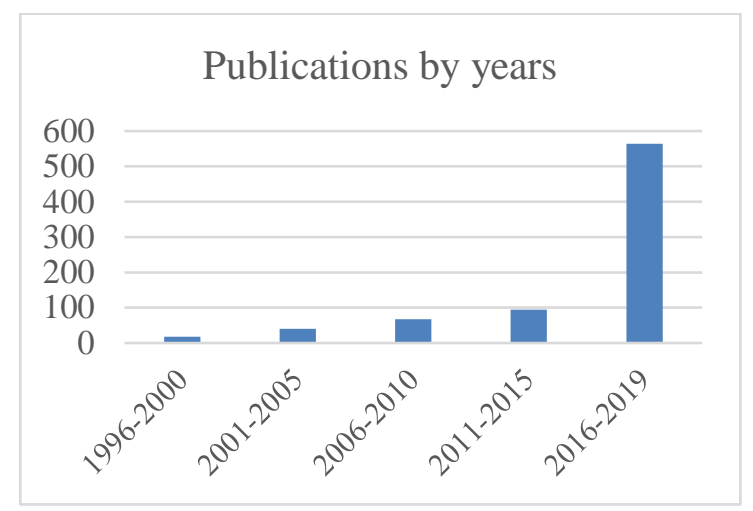

Figure 1. Publications using "collaborative robot*" or "cobot*" in the title or as a keyword, Web of Science (January 2020)

\subsection{Market interest}

Until recently, the global market value of cobots has remained relatively minor with an estimated global turnover of less than $\$ 600$ million in 2018 (Sharma, 2019). This, however, still represents an almost $50 \%$ growth rate compared to 2017. Cobots are particularly starting to make an impact in the largest market sector for industrial robots, the automotive industry (Bogue, 2016). Forecasts for the annual revenues of collaborative robots are highly optimistic, e.g. suggesting global revenues of \$7.6bn in 2027 (Sharma, 2019 ) or even more optimistically revenues of $\$ 9.2$ bn by 2025 (Murphy, 2017).

Expectations among market players are also high. Danish company Universal Robots sold the world's first commercial collaborative robot in December 2008. The company experienced a $72 \%$ growth rate in 2017 and expects to sustain $50-70 \%$ growth year-on-year for at least another five years (Crowe, 2018). It remains the largest global market player with a market share of more than 50 pct. of the global market; in 2018, the company also celebrated the sale of its $25,000^{\text {th }}$ cobot.

The supply of cobots was until recently limited to a handful of suppliers, but during the past few years, major industrial players such as ABB, Robert Bosch, and manufacturer of industrial robots KUKA have all joined the market for collaborative robots (Bogue, 2016). As the worldwide market rapidly expands, both market fragmentation and market selection mechanisms may come into play. For information on current market product offerings, see e.g. [insert some references].

\section{Cobots and collaboration}

The defining distinction between collaborative robots and traditional industrial robots is the direct interaction with human workers. Using this interaction enables organizations, at least in theory, to leverage the strengths and endurance of robots with the tacit knowledge and agile decision-making skills of humans. Thereby organizations benefit from the crucial advantages, which both humans and robots have (FastBerglund et al., 2016). Robots may ace repetitive and monotonous tasks, but human workers still handle unexpected and unplanned tasks better than their automated co-workers. In a sense, humans remain the most flexible resource in the system. By leveraging the heterogenous advantages, human-robot collaboration may be superior to purely robotic processes. This has also been shown to be true in experimental research settings (Bloss, 2016).
From

idea

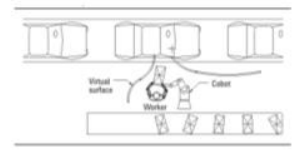

Peshkin \& Colgate (1999)
To practice

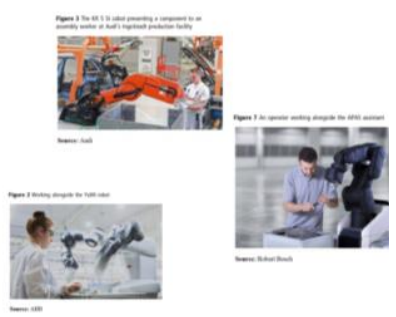

Bogue (2016)
Figure 2. Cobots from idea to practice

Drawing on El Zataari et al. (2019), the ways human operators and cobots interact can be defined as collaboration scenarios. Each scenario involves at least one human operator and at least one cobot sharing the same workspace in order to perform manufacturing process(es) on workpiece(s). Several definitions exist in literature distinguishing collaboration from cooperation or interaction. For example, Bendel (2018) notes that cooperation robots work with people step by step for a common goal, while collaborative robots work with people hand in hand on a common task. With a more lenient definition corresponding to the definition of cobot manufacturers, any robot operating alongside a human without a fence can be characterized as a collaborative robot El Zataari et al. (2019).

El Zataari et al. (2019) defines four distinct collaborative scenarios: 
- Independent: A human operator and a cobot work on separate workpieces, independently, and for their individual manufacturing processes. The collaborative element constitutes the shared workspace without cages or fences.

- Simultaneous: A human operator and a cobot operate on separate manufacturing processes at the same workpiece at the same time. Concurrently operating on the same workpiece minimizes transit time, improves productivity and space utilization, but as such, there is no time or task dependency between the humans and the cobot.

- Sequential: A human operator and a cobot perform sequential manufacturing processes on the same workpiece. Here, there are time dependencies between the processes of the operator and the cobot; often the cobots is assigned to handle the more tedious processes, which may also improve the operator's working condition.

- Supportive: A human operator and a cobot work on the same process on the same workpiece interactively. Here, there may ba full dependencies between the human and the cobot, as one cannot perform the task without the other.

At the present stage most examples of cobots deployed in industrial settings related to the 'independent' or the 'simultaneous' collaboration scenarios. Most advanced research projects, aiming to break new ground, can be categorized as striving for 'sequential' or 'supportive' collaboration scenarios though (El Zatari et al., 2019).

These scenarios demand more sophisticated systems and solutions. As the degree of interdependency and collaboration, cobots need to have improved semantic understandings of the task goal and the actions and intents of its human co-workers. Similarly, the human workforce needs to able to communicate with the cobot in intuitive ways.

\section{Cobots in the Industry 4.0-paradigm}

The usage of cobots supplements ongoing trends of automation manufacturing technology, through the socalled Industry 4.0. The Industry 4.0-paradigm promises the opportunity of achieving efficiency, cost reduction, increased productivity, and increased flexibility through integrated automation systems and Cyber-Physical Systems (Villani et al., 2018; Kadir et al., 2018). Manufacturing companies competitive in the future environment have to engage in more flexible production processes with mass customization and small batch sizes. Automation aids in some part, but traditional robotics do not always provide suitable solutions, as the manufacturing industry shifts from mass production to mass customization and towards agile manufacturingparadigms (Bloss, 2016; Dalle Mura \& Dini, 2019; Kootbally, 2016).

Industrial automation and classical robotic work cells lack versatility and flexibility, and high changeover times make it difficult to adapt to dynamic environments or to efficiently produce small-batch production (Kootbally, 2016; Villani et al., 2018). Agile manufacturing systems must be flexible, open, scalable and re-configurable, and lightweight, mobile cobots paired with the skills and the flexibility of humans deliver this more successfully.

A comparison between traditional industrial robots and cobots can be seen in Figure 3 below. The characteristics of collaborative industrial robots suit the demands of Industry 4.0 better.
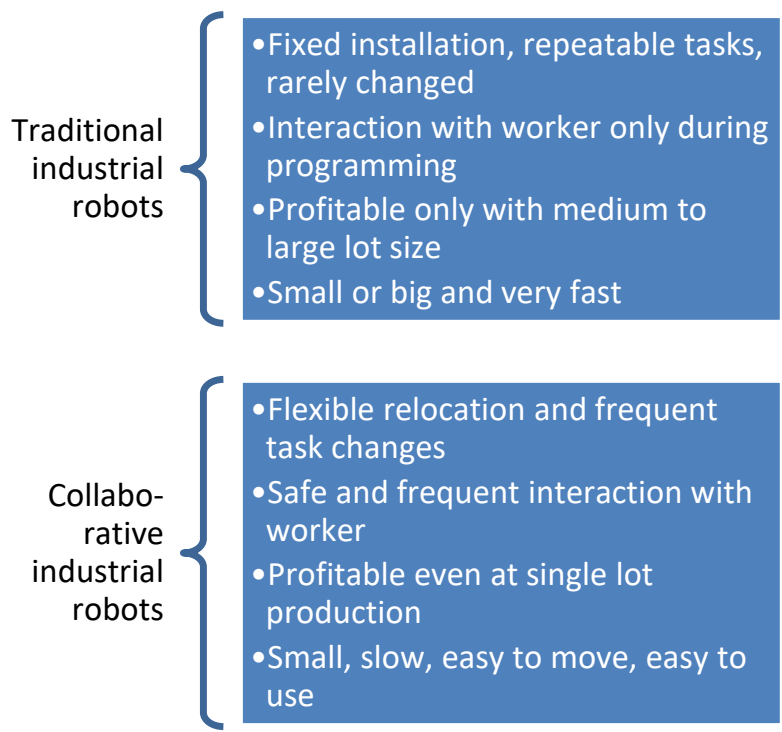

Figure 3. Characteristics of traditional industrial robots and of collaborative robots (modified from Villani et al., 2018)

\section{Cobots answers to megatrends}

The rapid technological development within the manufacturing industry is not the only megatrend, for which cobots may come to play a role.

The European Union's European Strategy and Policy Analysis System (ESPAS) foresee a range of Global Trends to 2030 (ESPAS, 2019), including climate change and environmental pressures, shrinking workforces and aging populations in Europe, and changing trade patterns on account of geopolitical shifts. Cobot developments may be shaped by as well as help shape the responses to these global trends. 


\subsection{Cobots as a response to climate change}

Recent research suggests that cobots and robotics, in general, can contribute to improved resource efficiency and work as important enablers of remanufacturing and circular economy and remanufacturing (Huang et al., 2019; Sarc et al., 2019).

\subsection{Cobots as a response to demographic shifts}

Since the original formulation of the cobot concept, ergonomics has been a major point of focus. Already the seminal article by Peshkin \& Colgate (1999) explicitly states that "Cobots can address ergonomic concerns, and provide productivity benefits as well.". Ergonomic concerns remain a strong argument cited in the literature for the introduction of cobots. More than $30 \%$ of manufacturing workers in Europe are affected by lower back pain, and initiatives addressing this are highly sought after (Cherubini et al., 2016; Maurice et al., 2017). A significant amount of research thus relates to the possibility of cobots to reduce work-related musculoskeletal disorders (MSDs) as well as stress and fatigue of operators. By reducing physical demands for workers, cobots can also yield more inclusive labor markets in which aging workers may be able to stay on for longer periods before retirement than now (Calzavara et al., 2020).

\subsection{Cobots as a response to geopolitical shifts}

As the geography of global manufacturing may shift with geopolitical trends, this could also affect future cobot trends. An explicit argument for political backing of Industry 4.0-initiatives, e.g. in Germany and the United States, has been to ensure the continued competitiveness of industries in high-cost manufacturing environments. According to this idea increased uptake of robotics and automation help 'bring manufacturing back' (Kaivo-oja et al., 2018; Ancarani et al., 2019). While the hypothesis is still being tested (see e.g. de Backer et al., 2018), in a case study of manufacturing business with cobot-investments the CEO does highlight the ability of the firm to remain competitive with outsourced competitors was highlighted as the main investment motivation (Kadir et al., 2018).

Having said that, there is little guarantee that classic high-cost manufacturing environments in OECD-countries will be the main markets for the uptake of cobots. There has for example also been recent talks of "China's 'cobot' revolution" (Halder, 2019), as growth in the Chinese market for cobots exceeds global developments. Cobots could, therefore, play a role in making the Chinese manufacturing economy even more competitive vis-a-vis high-cost competitors, and/or allow the country to keep its manufacturing market share even as wages and other costs may rise above e.g neighboring countries in Southeast Asia.

\section{Frontiers of current research: Glimpses of the futures of cobots}

In this section, we present a scan of the current frontiers of cobot research based on a horizon scanning of recent academic literature. The overview is presented in figure 4 below.
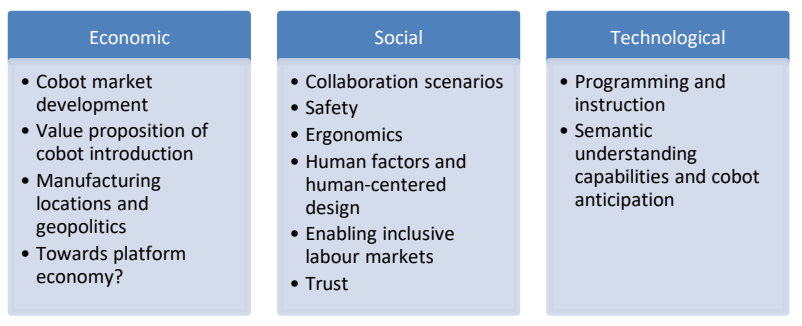

Figure 4. Three frontiers of cobot research (Knudsen \& Kaivo-oja, 2019)

\subsection{Economic dimensions}

As the cobot market grows worldwide, it could bring about major changes to the sector. As noted, larger industrial players have already appeared on the scene. The development of the market in terms of both suppliers and demand can affect what will become the dominant type of cobots as well as the dominant cobot markets. Markets in this sense must be understood both as geographic markets and as sectors of industrial manufacturing and beyond.

As with robots in general, there are practically no limits to where cobots can potentially be applied. During the next decade, we are likely to see cobots in healthcare, and education, as well as in manufacturing.

For this cobot development to take off, cobots must consistently prove themselves worthwhile in new settings. This most likely starts with increased exposure in industrial manufacturing settings. While cobots are more flexible and cheaper than traditional industrial robots, customers also here will still need to be able to justify their investment. Especially smaller SMEs could find themselves with challenges in looking for new tasks for cobots if original plans are altered (as documented by Kadir et al., 2018).

Another possible frontier is what the optimal business model for the cobot industry should look like. In recent years the global market leader Universal Robots have moved towards platforms business models. CTO and co-founder Esben Østergaard has envisioned the development as a 'dating service for problems and solutions' (Waurzuniak, 2016), and stressed how the company wants to underpin their customers' ability to customize their own solutions. This arguably mirrors the agile value proposition of cobots well. Business models of industrial robots today tend to be rather traditional, and Landscheidt et al. (2018) argue that business model change is necessary rather than just possible. 


\subsection{Social dimensions}

The sci-fi writer Isaac Asimov famously formulated his 'Three Laws of Robotics' in his 1942 short story Runaround (Murphy \& Woods, 2009):

1. "A robot may not injure a human being or, through inaction, allow a human being to come to harm. "

2. "A robot must obey the orders given by human beings except where such orders conflict with the First Law."

3. "A robot must protect its own existence as long such protection does not conflict with the First or Second Laws."

The 'laws' clearly reflect how the safety of humans is the foremost concern in Asimov's vision of humanrobot interaction. As might be expected, this view is still prevalent in robotics research to this day, and after a series of unfortunate robot-related events, guidelines for robot safety began to emerge in the mid-1980s (Marvel $\&$ Norcross, 2017). In general, traditional safety-related research focused on maintain operator safety by separating active machinery and robots from the workforce. Duffy (2016) notes that even recent books on occupational health and safety place the emphasis regarding industrial robots on preventing interaction between humans and robots.

However, recent updates to robot safety standards do address new co-working scenarios (Villani et al., 2018). The international ISO-standard identifies four collaborative modes: i. Safety-rated monitoring stop (SMS), ii. Hand guiding (HG), iii. Speed and separation monitoring (SSM), and iv. Power and force limiting (PFL). As collaborative robotics progresses, research on safety issues within each of these four collaborative modes must simultaneously progress.

It has been rightfully argued that safety requirements should not be seen as a hindrance to cobot performance, instead performance should be optimized subject to the constraint of safety, while safety solutions must also take needs of vulnerable users into account and acknowledge different skills and capabilities (Villani et al., 2018).

This also entails the inclusion of human factors in the thinking on cobots, as well as pursuing the previously mentioned utilization of cobots in order to address ergonomic and labor market inclusion concerns.

Another key element of the social dimension relates to modes of cooperation and collaboration with workers. As new tasks also increasingly involve interaction with computational devices, future shopfloor factory workers take over operations, which previously have been engineering tasks (Stern \& Becker, 2017). Smart and skilled operators collaborate with robots and are aided by machines, advanced human-machine interaction technologies and adaptive automation (Mattson et al., 2020).

\subsection{Technological dimensions}

As the cobot development is so rapid, the technological frontiers are continuously moved. Across the literature, two main research themes can, however, be detected. In one thread, academics try to push the limits for programming and instructions of cobots in order to simplify this. The other thread aims to produce more advanced or complex forms of interaction by enabling cobots with semantic understanding capabilities or AI-aided anticipation skills.

An important challenge for exploiting collaborative robots in a fast-paced dynamic workflow is instruction and programming of the cobot. Rather than remove the need for human interventions, the current trends see a change of tasks and the persons involved in daily operation procedures. Traditional robot programming has been the role of engineers, but in order to exploit reduced change-over times and increased efficiency, the task of programming is moving to operators on the shop floor (Schou et al., 2018). This has both monetary benefits (decreasing the need for high-wage engineering), it simultaneously allows the operator to channel valuable process knowledge and experience into the instruction.

However, the change magnifies the demands for programming of the cobot to be simple and intuitive. As a cobot CTO stated, their mission is put the control of robots back into the hands of the operator, and that 'If you can work a smartphone, you can program this robot' (Waurzuniak, 2016).

Another key theme in the on-going robot and cobot research is how to achieve more advanced forms of interactions (Ivaldi, 2018). Ideally, future cobots can take into account human cues, movements, and intentions with the ability to distinguish between workrelated intentional and non-intentional human gestures. It is a requirement for natural human-robot collaboration that the robot is endowed with the capability to capture, process, and understand accurately and robustly human requests (Maurtua et al., 2017). This means endowing robots with semantic understanding capabilities; a very challenging task, but also a very important one. Research related to human-robot collaboration also revolves around enhancing particular enabling functions like visual perception and action recognition which enables human awareness and promotes flexible cobot behavior (El Zaatari et al., 2019).

In order to provide optimal physical assistance to humans, it has been argued, cobots also need to be able to predict their intent, future behavior, and movements (Ivaldi, 2018). Recognizing the use of force/pressure sensors in contact to anticipate the movements and 
objectives of the human partner, represents an alternative approach to the design of human-robot interfaces (Aroudani et al., 2018). Implementation of predictive and adaptive control methodologies is still in a premature state, and it also raises new questions such as whether robot adaptation should be generic or userspecific.

Ma et al. (2019) observe that as more and more machines are used in manufacturing, the onus of humanmachine interaction has gradually shifted from the adaption of humans to machines to the adaption of machines to humans. This observation also neatly captures and summarizes many aspects of the technological research frontier for cobots, as described in the previous paragraphs.

\section{Conclusions}

As shown in the article, there is significant interest in the topic of collaborative robots. The market for cobots in the manufacturing industry is growing, and the academic literature on the topic has expanded rapidly during the past few years. Neither in the literature nor elsewhere have we detected any signals that this trend is likely to be broken. Instead, it seems probable that cobot automation will be at the center stage of the humanrobot interaction in the 2020s.

Cobots enables, at least in theory, optimal combinations of the skills of machines with the skills of humans. This combination of the robot's ability to perform repetitive tasks and handle the physical workload with the tacit knowledge and adaptational capabilities of humans can bring promising results. As shown, the collaboration also fits within new agile manufacturing paradigms with increased customization and smaller batch sizes. In many ways, cobots are better equipped to take part in an 'Industry 4.0' than traditional industrial robots.

The brief overview also gives hints of future developments. We can see that there are current research frontiers related to both economic, social, and technological dimensions. The cobot industry is still in many ways in its infancy and questions of business models, value propositions and customers remain in flux. The social dimension relates e.g. to the various collaboration scenarios of humans and machines; a topic of great current interest across many academic disciplines. Technological trends focus on making the programming, instruction, and use of cobots simpler in order to maximize benefits, as well as endowing new generations of cobots with increasingly complex capabilities of understanding, intelligence, and prediction.

Together, the economic, social and technological frontiers show important trendlines that will help to shape the futures of cobots and cobot uptake.

\section{Acknowledgments}

The authors wish to thank the Finnish Strategic Research Council for providing funding for the project Manufacturing 4.0 [grant agreement 313395], and the European Regional Development Fund, under a grant agreement with the Research Council of Lithuania, for providing funding for the project "Platforms of Big Data Foresight (PLATBIDAFO)" (project No. 01.2.2LMT-K-718-02-0019).

\section{References}

Ancarani, A., Di Mauro, C., \& Mascali, F., 2019. Backshoring strategy and the adoption of Industry 4.0: Evidence from Europe. Journal of World Business, 54(4), 360-371.

Aroudani A., Zanchettin, A. M., Ivaldi, S., AlbuSchäffer, A., Kosuge, K., \& Khatib, O., 2018. Progress and prospects for human-robot collaboration. Autonomous Robots, 42(5), 957-975.

Bendel, O., 2018. Co-robots from an Ethical Perspective. In Dornberger, R. (ed.), Business Informations Systems and Technology 4.0: New Trends in the Age of Digital Change. Springer, Cham.

Bloss, R., 2016. Collaborative robots are rapidly providing improvements in productivity, safety, programming ease, portability and cost while addressing many new applications. Industrial Robot: An International Journal, 43(5), 463-468.

Bogue, R., 2016. Europe continues to lead the way in the collaborative robot business. Industrial Robot: An International Journal, 43(1), 6-11.

Calzavara, M., Battini, D., Bogataj, D., Sgarbossa F., Zennaro, I., 2020. Ageing workforce management in manufacturing systems: state of the art and future research agenda. International Journal of Production Research, 58(3), 729-747.

Cherubini, A., Passama, R., Crosnier, A., Lasnier, A., \& Fraisse, P., 2016. Collaborative manufacturing with physical human-robot interaction. Robotics and Computer-Integrated Manufacturing, 40, 1-13.

Crowe, S., 2018. Universal Robots Sees Strong Growth in Asia. The Robot Report, March 12, 2018. Accessed 27.01.2020 at https://www.therobotreport.com/universal-robotsstrong-growth-asia/

Dalle Mura, M., \& Dini, G., 2019. Designing assembly lines with humans and collaborative robots: A 
genetic approach. CIRP Annals - Manufacturing Technology, 68(1), 1-4.

de Backer, K., DeStefano, T., Menon, C., Suh, J. R., 2018. Industrial robotics and the global organisation of production. OECD Science, Technology and Industry Working Papers, 2018/03.

Djuric, A. M., Urbanic, R., Rickli, J. R., 2016. A Framework for Collaboarative Robot (CoBot) Integration in Advanced Manufacturing Systems. SAE International Journal of Materials and Manufacturing, 9(2), 457-464.

Duffy V. G. (2016). Modern Human-Robot Interaction in Smart Services and Value Co-Creation. In V. G. Duffy (Ed.), Digital Human Modeling: Applications in Health, Safety, Ergonomics and Risk Management. Springer International Publishing.

El Zataari, S., Marei, M., Li, W., Usman, Z., 2019. Cobot programming for collaborative industrial tasks: An overview. Robotics and Autonomous Systems, 116, 161-180.

European Strategy and Policy Analysis System (ESPAS), 2019. ESPAS Report 2019: Global Trends to 2030. Accessed at 27.01.2020 at https://espas.secure.europarl.europa.eu/orbis/sites/d efault/files/generated/document/en/ESPAS_Report2 019.pdf

Fast-Berglund, Å., Palmkvist, F., Nyqvist, P., Ekered, S., Åkerman, M., 2016. Evaluating Cobots for Final Assembly. Procedia CIRP, 44, 175-180.

Goldberg, K., 2019. Robots and the return to collaborative intelligence. Nature Machine Intelligence, 1, 2-4.

Halder, B., 2019. How China's 'Cobot' Revolution Could Transform Automation. Ozy. Accessed 27.01.2020 at: https://www.ozy.com/fastforward/how-chinas-cobot-revolution-couldtransform-automation/93044/

Hentout, A., Aouache, M., Maoudj, A., \& Akli, I. 2019. Human-robot interaction in industrial collaborative robotics: A literature review of the decade 20082017. Advanced Robotics, 33(15-16), 764-799.

Huang, J., Pham, D. T., Wang, Y., Qu, M., Ji, C., Su, S., Xu, W., Liu, Q., Zhou, Z., 2019. A case study in human-robot collaboration in the disassembly of press-fitted components. Proceedings of the Institution of Mechanical Engineers, Part B: Journal of Engineering Manufacture.
Ivaldi, S. (2018). Intelligent Human-Robot Collaboration with Prediction and Anticipation. ERCIM News.

Kaivo-oja, J., Knudsen, M. S., \& Lauraeus, T., 2018. Reimagining Finland as a Manufacturing Base: The Nearshoring Potential of Finland in an Industry 4.0 Perspective. Business, Management \& Education, $16,65-80$.

Kadir, B. A., Broberg, O., Souza de Conceicao, C., 2018. Designing human-robot collaborations in industry 4.0: explorative case studies. DS92: Proceedings of the DESIGN 2018 15th International Design Conference, 601-610

Knudsen, M. S., Kaivo-oja, J., 2019. Out of the Cages: Here comes the cobots. Tulevaisuuden tutkimuskeskuksen blogi. Accessed 27.01.2020 at: https://ffrc.wordpress.com/2019/11/05/cobots/

Kootbally, Z., 2016. Industrial robot capability models for agile manufacturing. Industrial Robot: An International Journal, 43(5), 481-494.

Korn, O., Bieber, G., Fron, C.., 2018. Perspectives on Social Robots: From the Historic Background to an Expert's View on Future Developments. PETRA '18 Proceedings of the $11^{\text {th }}$ PErvasive Technologies Related to Assistive Environments Conference.

Landscheidt, S., Kans, M., Winroth, M., \& Wester, H., 2018. The future of industrial robot business: Product or performance based? Procedia Manufacturing, 25, 495-502.

Ma, X., Tao, F., Zhang, M., Wang, T., Zuo, Y., 2019. Digital twin enhanced human-machine interaction in product lifecycle. Procedia CIRP, 83, 789-793.

Marvel, J., 2014. Collaborative Robots: A gateway info factory automation. National Institute of Standards and Technology. Accessed 27.01.2020 at https://tsapps.nist.gov/publication/get_pdf.cfm?pub _id $=916485$

Marvel, J. A., Norcross, R., 2017. Implementing speed and separation monitoring in collaborative robot workcells. Robotics and Computer-Integrated Manufacturing, 144-155.

Mattson , S., Fast-Berglund, Å., Li, D., \& Thorvald, P. (2020). Forming a cognitive automation strategy for Operator 4.0 in complex assembly. Computers \& Industrial Engineering, 139..

Maurice, P., Padois, V., Measson, Y., Bidaud, P., 2017. Human-oriented design of collaborative robots. 
International Journal of Industrial Ergonomics, 88102.

Maurtua, I., Fernandez, I., Tellaeche, A., Kildal, J., Susperregi, L., Ibarguren, A., Sierra, B., 2017. Natural multimodal communication for humanrobot collaboration. International Journal of Advanced Robotic Systems, 1-12.

Murphy, A., 2017. Industrial: Robotics Oulook 2025. Loup Ventures. Accessed 27.01.2020 at: https://loupventures.com/industrial-roboticsoutlook-2025/

Murphy, R. Woods, D.D., 2009. Beyond Asimov: The Three Laws of Responsible Robotics. IEEE Intelligent Systems, 24(4), 14-20.

Peshkin, M., Colgate, J. E., 1999. Cobots. Industrial Robot: An International Journal. 26(5), 335-341.

Sarc, R. Curtis, A., Kandlbauer, L., Khodier, K., Lorber, K.E., Pomberger, R., 2019. Digitalisation and intelligent robotics in value chain of circular economy oriented waste management - a review. Waste Management. 95, 476-492.

Schou, C., Andersen, R. S., Chrysostomou, D., Bøgh, S., Madsen, O., 2018. Skill-based instruction of collaborative robots in industrial settings. Robotics and Computer Integrated Manufacturing, 72-80.

Sharma, A, 2019. Cobot Market Outlook Still Strong, Says Interact Analysis. Robotics Business Review. Accessed 27.01.2020 at: https://www.roboticsbusinessreview.com/manufact uring/cobot-market-outlook-strong/

Stern H., Becker, T., 2017. Development of a Model for the Integration of Human Factors in Cyber-Physical Production Systems. Procedia Manufacturing, 9, 151-15

Villani, V., Pini, F., Leali, F., \& Secchi, C., 2018. Survey on human-robot collaboration in industrial settings: Safety, intuitive interfaces and applications. Mechatronics, 55, 248-266.

Waurzuniak, P., 2016. New Collaborative Robot App Store Helps Users Find Solutions to Automation Problems. Manufacturing Engineering, 18-22. 